\title{
Beneficios y retos de la enseñanza del trabajo de campo en el campo: el caso del posgrado en antropología social de la Universidad Iberoamericana, ciudad de México
}

\author{
Roger Magazine
}

\begin{abstract}
El posgrado en antropología de la Universidad lberoamericana capacita a sus estudiantes para la investigación mediante el modelo de Palerm de enseñar el trabajo de campo en el campo.Varios aspectos de este curso se adaptan a las demandas de la institución, a particularidades regionales y a los intereses de los investigadores, pero las premisas básicas permanecen: la enseñanza práctica, la exposición de los estudiantes a las investigaciones de los profesores y los temas de investigación surgidos de la realidad observada en el campo. En este artículo discutiré los beneficios y desventajas de esta formación y la compararé con modelos más individualizados que predominan en Estados Unidos y en la disciplina en general. Exploro los retos que enfrentan profesores y alumnos para mantener esta práctica al encarar recortes presupuestales, exigencias de eficiencia terminal y de incremento de la producción académica.
\end{abstract}

PALABRAS CLAVE: trabajo de campo, docencia del posgrado, Universidad Iberoamericana, Ángel Palerm, antropología mexicana

\section{The Benefits of and Challenges to Fieldwork Training in the Field: The Case of the Social Anthropology Graduate Program at the Universidad Iberoamericana in Mexico City}

The Universidad lberoamericana's graduate program in anthropology trains its students through Palerm's model of teaching fieldwork in the field. Several aspects of this course are adapted to institutional demands, regional particularities and individual interests, but the basic premises remain: fieldwork taught in practice, students exposed to professors' research, and dissertation topics that emerge from realities observed in the field. In this article I discuss benefits and disadvantages of this training for students and faculty, and compare it to more individualized models currently dominant in United States and in the discipline in general. I also explore challenges confronted by professors and students to maintain this off-campus instruction in the face of budget cuts and pressures to accelerate the rate of graduate training and the academic production.

KEYWORDS: fieldwork, graduate instruction, Universidad Iberoamericana, Ángel Palerm, Mexican anthropology

Roger Magazine: Universidad Iberoamericana, Distrito Federal, México roger.magazine@ibero.mx

Traducción: Jaime Sanromán Ruiz

Desacatos, núm. 45, mayo-agosto 2014, pp. 27-34

Recepción: 9 de noviembre de 2012 / Aceptación: 25 de noviembre de 2013 


\section{INTRODUCCIÓN}

$\mathrm{E}$ mpezaré con una descripción de la forma en que se enseña el trabajo de campo en la actualidad a los estudiantes del posgrado en antropología de la Universidad Iberoamericana (UIA), en la ciudad de México. A continuación, compararé esta formación con la que se recibe en Estados Unidos y tomaré como ejemplo mi propia experiencia durante el posgrado. También discutiré algunos de los beneficios y limitaciones de cada tipo de formación e intentaré exponer las principales diferencias entre ellos. Concluyo con una breve discusión sobre un par de amenazas para la continuidad de la enseñanza del trabajo de campo en el campo en México.

\section{LA ENSEÑANZA DE LA INVESTIGACIÓN A TRAVÉS DE “LA PRÁCTICA DE CAMPO”}

Tras su primer año de cursos teóricos en los programas de maestría o doctorado de la UIA, se espera que los estudiantes de antropología participen a fines de mayo en lo que se denomina "una práctica de campo supervisada", que dura seis semanas. De los ocho académicos del programa de la UIA, usualmente tres o cuatro llevan grupos de tres a 12 estudiantes a una región rural, donde ellos mismos tienen un proyecto de investigación, para dirigir una práctica. En los últimos años, los profesores han llevado a sus alumnos a diferentes partes del país, como Chiapas, Oaxaca, Campeche y Tlaxcala. Usaré como ejemplo la práctica que he dirigido durante varios veranos desde 2001 en la región de Texcoco, en el Estado de México, donde Ángel Palerm comenzó a formar a sus estudiantes en la década de 1960. Él y su esposa y colega Carmen Viqueira compraron una casa en el pueblo de Tepetlaoxtoc para que fuera la estación de campo para las prácticas en la región de Texcoco. Eventualmente, la UIA compró la casa y continuamos usándola como estación base para la práctica.
Cuando empecé a trabajar como profesor en la UIA en 1999, todos mis colegas habían sido discípulos de Palerm y todos habían participado en una "práctica de campo", aunque sólo dos de ellos lo habían hecho directamente bajo su supervisión. Otros habían sido entrenados en el campo por los primeros alumnos de Palerm o por Hugo Nutini, como en el caso de mi colega David Robichaux cuando estudiaba la maestría en la UiA. A partir de las instrucciones de mis colegas, que siempre se referían a "cómo Ángel hacía las cosas", y de mi propia participación durante el 2000 en la práctica supervisada por Robichaux en la zona rural de Tlaxcala, aprendí cómo supervisar la práctica. Desde entonces, los catedráticos que se han unido al posgrado han aprendido el modelo de manera similar: a través de la instrucción informal con los colegas y en las prácticas dirigidas por otros profesores. Desde luego, cada profesor innova el modelo al incorporar elementos de su propia formación e intereses. Las particularidades regionales, como tamaño, rutas de transporte, integración regional y otros factores, también tienen influencia en el modo específico en que se lleva a cabo cada práctica. Aunque las prácticas varían de profesor a profesor, de año a año y de región a región, prevalece un esfuerzo por mantener algunos de los principios y procedimientos centrales, que mencionaré más adelante.

El profesor y los estudiantes pasan la primera semana juntos como grupo y, en el caso de la práctica en la región de Texcoco, viven juntos en la estación de campo. Cada mañana el grupo visita dos o tres pueblos de la región. Los estudiantes los recorren en grupos de dos a cuatro, se detienen para charlar con la gente que encuentran, les hacen preguntas no delicadas sobre la comunidad, sus tradiciones y costumbres. También solicitan información sobre posible alojamiento para uno o dos estudiantes durante una estancia de cinco semanas. Incluso si a ellos no les interesa quedarse en un determinado pueblo, siempre buscan orientación respecto del alojamiento potencial para sus compañeros. Estas visitas iniciales durante la primera semana exponen a los 
alumnos a la experiencia de abordar a los extraños y ganar su confianza. Además, les dan oportunidad de familiarizarse con la región como un entorno mayor, seleccionar el pueblo en el que desean llevar a cabo su estudio de cinco semanas y hallar un lugar donde alojarse.

$\mathrm{Al}$ volver a la estación, los estudiantes pasan la tarde escribiendo sus diarios de campo. Al anochecer el grupo se reúne y los estudiantes leen una parte de sus diarios, reciben comentarios de sus compañeros y del profesor o profesores. Algunos de los problemas más comunes con los diarios de campo durante los primeros días son: 1) Alcanzar el nivel adecuado de abstracción. Algunos estudiantes tienden a perderse en los detalles al grado de que no acaban de escribir, mientras que otros omiten muchos detalles o hechos completos, lo que deja las notas inconclusas. Ambos problemas se relacionan con entender qué es "un dato" para el antropólogo. 2) La tendencia de los estudiantes de calificar o juzgar sus hallazgos o de elaborar interpretaciones prematuras. Este problema se advierte en el uso de adjetivos como "grande", "urbano", "rico" que tienen el potencial de causar confusión entre el entendimiento propio del estudiante y la realidad local.

Al final de la primera semana, los estudiantes, a menudo con el profesor, vuelven al pueblo en el que quieren realizar su investigación y tratan de concretar el asunto del alojamiento. Idealmente reciben una invitación para permanecer con alguna familia - gratis o pagando renta-, aunque a veces aceptan dormir en edificios públicos, como un centro de

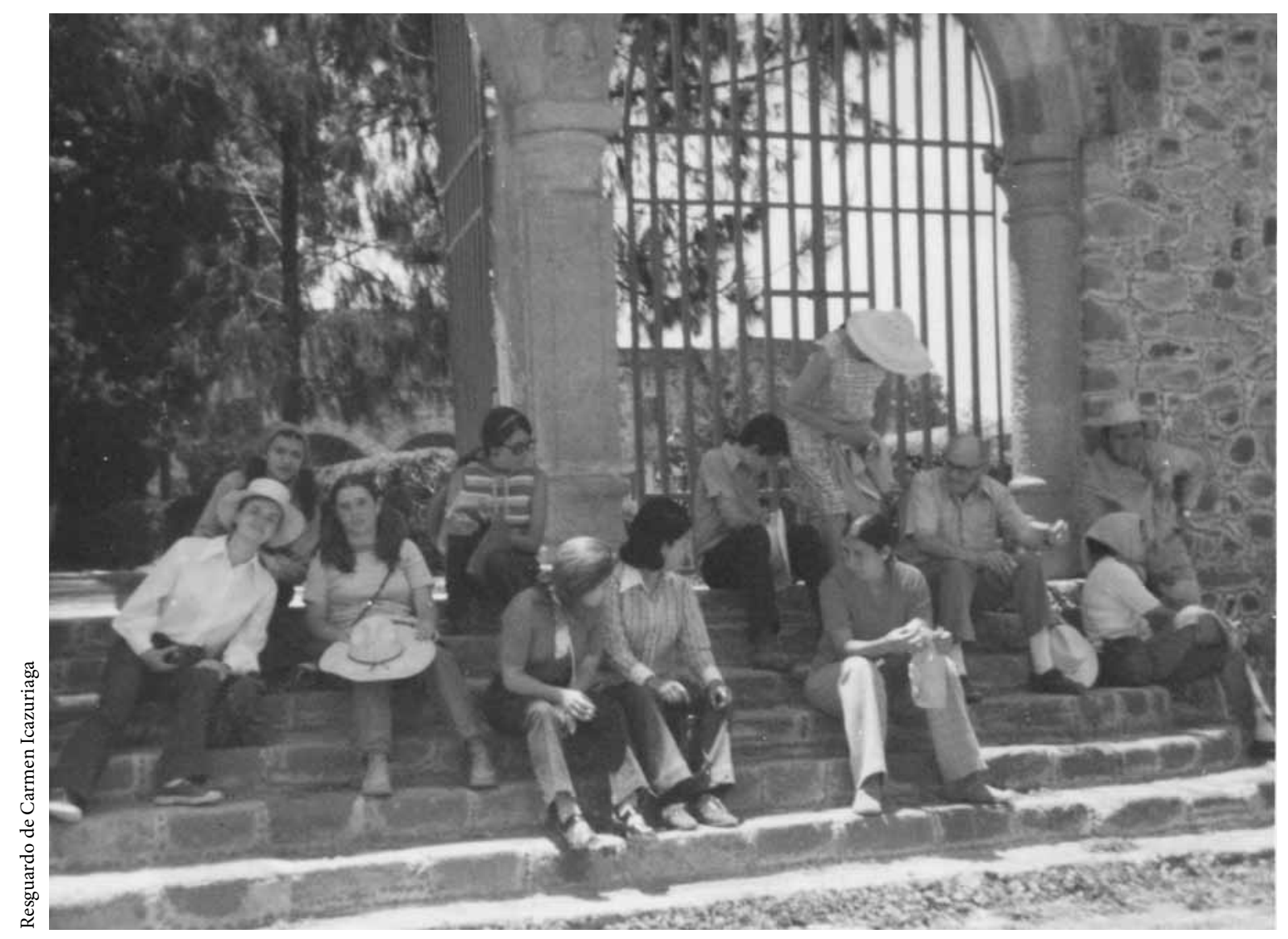

Ángel Palerm y estudiantes en la iglesia de Papalotla, Estado de México, ca. 1971. 
salud o la oficina local de gobierno. Se permite un máximo de dos alumnos en la misma localidad. En los recorridos se evita visitar los pueblos donde algún estudiante más avanzado está llevando a cabo investigación para su tesis, de modo que los nuevos estudiantes no interfieran con su labor. Al describir la práctica en la región de Texcoco, algunas colegas de otras instituciones hacen la observación de que los lugareños deben estar acostumbrados a recibir antropólogos después de casi 50 años de prácticas en la zona. Sin embargo, éste parece no ser el caso. Sólo de cuando en cuando encontramos residentes que recuerdan haber recibido o conocido a algún estudiante de la UIA. De hecho, cuando los habitantes escuchan que somos universitarios que llevan a cabo una "práctica", suponen que provenimos de la Universidad Autónoma Chapingo, una institución localizada en la región especializada en temas agrícolas, pecuarios y forestales. A diferencia de zonas más aisladas donde los antropólogos destacan como fuereños, en la región de Texcoco nos mezclamos que vive o trabaja allí. Al considerar todos los cambios que ocurren en el área y lo que aún no acabamos de comprender por completo, argumentaría que ésta no ha sido ni está sobreestudiada.

Durante las siguientes cinco semanas los estudiantes realizan una investigación en los pueblos en que se alojan y el grupo entero se reúne una vez a la semana en la estación de campo para presentar y discutir sus hallazgos, y para aprender nuevas técnicas de investigación. Cada semana los estudiantes se enfocan en un tema particular, el cual se revisará en la siguiente reunión. Para la primera semana, los estudiantes elaboran un mapa del pueblo basado en sus recorridos, observaciones propias e indicaciones de los oriundos. Además, se espera que describan, al menos, dos casas por dentro y por fuera. El mapa y estas descripciones son un fin en sí mismos, pero también sirven de pretexto para que los estudiantes comiencen a recorrer el pueblo y a charlar con los residentes con la intención de identificar

participantes - informantes - potenciales y hacer que la gente se acostumbre a su presencia y empiece a tenerles confianza. Durante la segunda semana los estudiantes se enfocan en las actividades económicas, incluyendo la agricultura. La siguiente investigan sobre la organización comunitaria, el gobierno local, los puestos civiles y religiosos, llamados "cargos", y las celebraciones comunales, en particular las fiestas patronales. Dedican la cuarta semana a temas de parentesco, organización familiar y patrones de residencia, ejercicio para el cual deben elaborar genealogías completas de dos familias. En la quinta semana realizan un pequeño censo de 25 casas, lo que les da oportunidad de practicar esta técnica y, de nuevo, da pie a tocar más puertas y conocer a otros participantes potenciales. En algún momento durante las cinco semanas los estudiantes habrán de acercarse a las escuelas locales y solicitar permiso para aplicar una encuesta a los alumnos. Todas estas técnicas - genealogías, censo y encuesta- son ejercicios: la experiencia de aprender la técnica al llevarla a cabo en la práctica es lo importante, más que el "éxito" al recopilar datos. El objetivo de un enfoque en estos temas básicos es alentar a los estudiantes a iniciar una investigación con una perspectiva amplia y holística, pero por ningún motivo se espera que limiten sus conversaciones y la recopilación de datos a éste. Al contrario, se les instruye a dejar que los temas fluyan naturalmente para aprender qué es importante para la gente del pueblo. Cada alumno es libre de investigar los temas particulares que le interesan.

En las reuniones semanales los estudiantes hacen una presentación de sus actividades de la semana y reciben comentarios y sugerencias de sus compañeros y del profesor. Los señalamientos del docente se dirigen a las actividades del alumno en campo, a la presentación en sí o al análisis o relevancia de sus datos. Los relativos a las presentaciones en sí buscan ayudarle a equilibrar los planteamientos generales con los casos o ejemplos concretos. Esto es, los comentarios tienen el objetivo de ayudarle a evitar 
generalizaciones sin fundamento por un lado, $\mathrm{y}$ a ir más allá de una simple lectura del diario de campo por el otro. Los relacionados con el análisis y la relevancia de los datos surgen por lo general de los intereses del profesor dentro de su propio proyecto de investigación en la región. En estos momentos podríamos decir que el aprendizaje práctico del estudiante emerge de su participación en un proyecto colaborativo de investigación y no simplemente de una práctica. El estudiante se beneficia al observar cómo el profesor analiza y encuentra significado a los datos presentados, mientras que éste aprende de los casos expuestos por los estudiantes. Al final de la reunión, el profesor enseña las técnicas de investigación que los estudiantes aplicarán la siguiente semana.

Al finalizar las seis semanas de la práctica, los estudiantes deben escribir un reporte etnográfico con sus hallazgos. Usualmente, dedican una sección a cada tema semanal: comienzan con una perspectiva regional basada en los recorridos de la primera semana y en los datos compartidos con sus compañeros que trabajaron en distintas comunidades. La sección final del reporte consiste en la formulación de preguntas y temas de investigación a futuro derivados de sus hallazgos. Esta formulación es, como el resto de la práctica, tomada como un ejercicio, aunque muchos estudiantes escriben su tesis de maestría o doctorado sobre éstos $\mathrm{u}$ otros temas relacionados en el mismo pueblo. Los alumnos que deciden volver al lugar para su investigación de tesis tienen la ventaja de no empezar de cero. Sin embargo, aun aquellos estudiantes que prefieren trabajar otro tema en un lugar distinto salen de la práctica con una comprensión cabal de los beneficios de plantear un tema de investigación con base en realidades concretas y en la experiencia de saber cómo hacerlo. Es importante mencionar que se les recuerda que consideren la práctica como una breve introducción a la vida en la comunidad y como un periodo demasiado corto para tener un entendimiento claro o completo de los procesos y prácticas locales.

\section{UNA MIRADA COMPARATIVA A LA ENSEÑANZA DEL TRABAJO DE CAMPO}

Ahora compararé de manera breve este método de enseñanza del trabajo de campo a los alumnos del posgrado de la Uia con la formación que recibí como estudiante de posgrado en la Universidad Johns Hopkins en Baltimore, Maryland, Estados Unidos, a mediados de la década de 1990. Hago esta particular comparación porque es lo que conozco por propia experiencia y porque creo que es similar a las de muchos alumnos que han estudiado en años recientes en programas de posgrado en instituciones en los Estados Unidos y otros países.

La única instrucción relacionada directamente con el trabajo de campo que recibí fue en un curso de un semestre dedicado a este tema e impartido en un aula de la Universidad. Y este curso se impartió sólo después de que mis compañeros y yo lo solicitáramos de manera insistente, porque no se incluía en el currículo. En su defensa, mis profesores argumentaban que los métodos de trabajo en campo no podían ser enseñados, queriendo decir, quizá, que no podían enseñarse en un aula. También realicé un verano - diez semanas - de trabajo de campo preliminar solo y sin supervisión, salvo por un par de cartas que intercambié con mi tutor. Conforme a la normatividad del programa, lo hice fuera de mi país de origen, Estados Unidos. Tuve la oportunidad de escoger dónde llevar a cabo la investigación y el tema. Fui a un país donde ninguno de mis profesores había trabajado antes, República Dominicana - aunque dos de ellos sí habían hecho investigación en otros países del Caribe-, y me ocupé de un tema desconocido para ellos: las ligas de beisbol para niños. Tras este trabajo de campo preliminar tomé una decisión individual al proponer la ciudad de México para desarrollar la investigación de mi tesis de doctorado. Me basé en algunas observaciones hechas durante una visita de tres semanas a México, en ideas derivadas de mi trabajo en República 


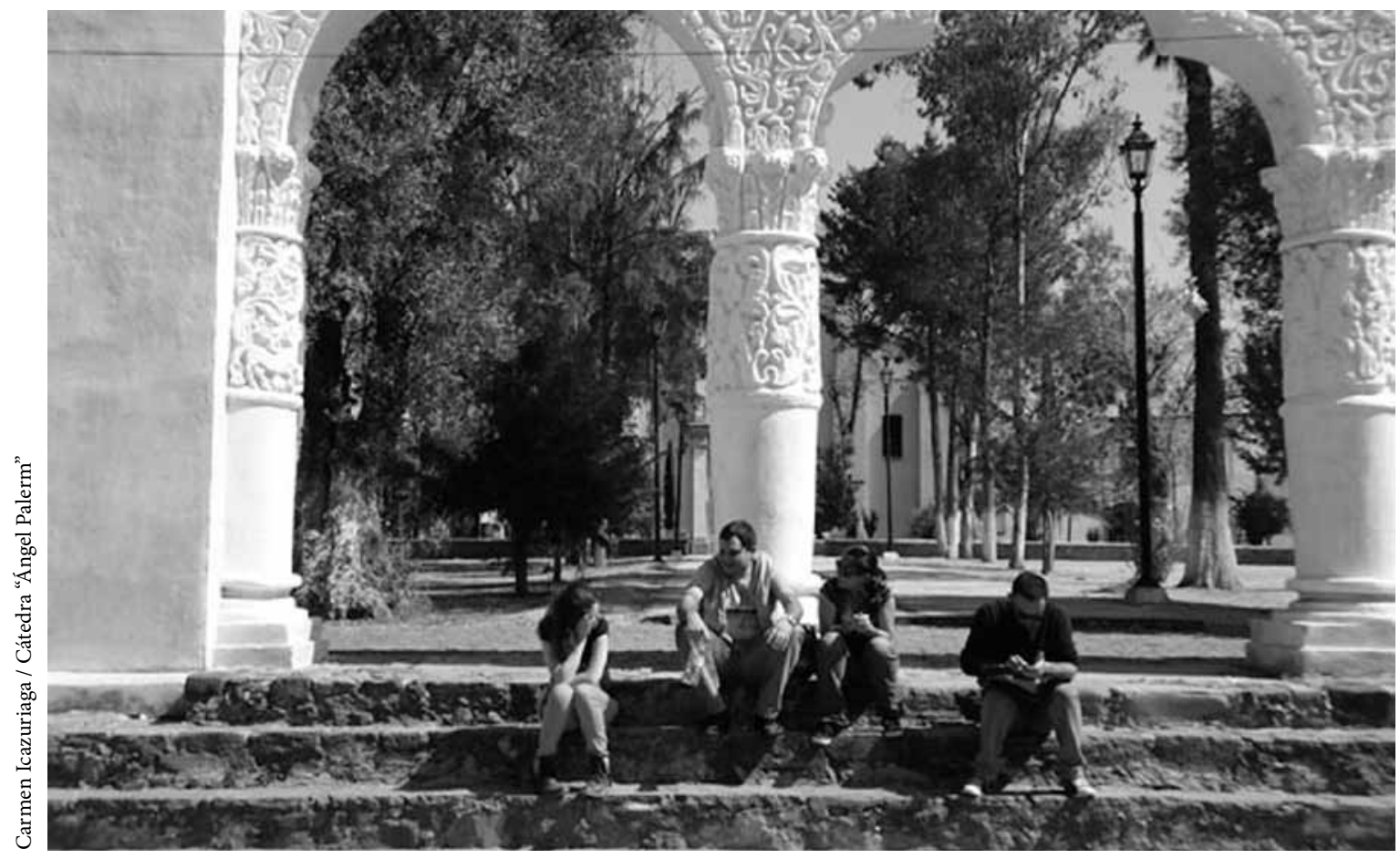

Breve descanso en el pórtico de la iglesia de Papalotla, Estado de México. Serie: Lectura del paisaje: Sistemas de riego en el Acolhuacan septentrional, 2014.

Dominicana y en lo que conocí de la bibliografía antropológica sobre el México urbano. Ninguno de mis profesores había realizado tampoco investigación en México ni en el tema que propuse estudiar allí: los aficionados al futbol profesional. Entre el curso sobre métodos y las conversaciones casuales, de alguna manera aprendí lo suficiente para llevar a cabo la investigación de campo y para escribir el diario. No obstante, mi trabajo no careció de deficiencias. Por ejemplo, no fue sino hasta la mitad de mi estancia en el campo cuando una profesora inquirió si codificaba mi diario por temas o usaba tarjetas indexadas. Fue la primera vez que escuché sobre esta metodología de las notas de campo y empecé a emplear un sistema de esta naturaleza. Además, no eché mano de métodos más allá de la observación participante y las entrevistas, como las genealogías o un censo, que podrían haber contribuido con otro tipo de datos a la investigación.
En comparación con la formación de trabajo de campo que los estudiantes de posgrado de la UIA reciben, la mía luce pobre. Sin embargo, logré producir resultados, lo cual quizá apoya el argumento de mis profesores acerca de que el trabajo de campo no puede enseñarse. No obstante, considero que mi investigación hubiera sido mejor $-\mathrm{y}$ sin duda me hubiera causado menos ansiedad - de haber tenido oportunidad de aprender con un profesor algunas de las técnicas del trabajo de campo en un entorno práctico. ¿A qué se deben estas diferencias entre la enseñanza del trabajo de campo en la UIA y la que recibí en Estados Unidos? Una explicación obvia es la diferencia entre la antropología de México y la de Estados Unidos en términos geográficos. Los antropólogos mexicanos, por lo general, estudian temas de México. Así, sus sitios para la investigación en campo se encuentran más cerca de sus lugares de residencia y de trabajo académico y hay una 
mayor concentración de antropólogos en estos sitios. En contraste, la antropología estadounidense por lo general lleva a cabo su investigación fuera del país, lo que conduce a una mayor dispersión de profesores y estudiantes en distintas y distantes partes alrededor del mundo. ${ }^{1}$

Otro factor que podría ayudar a explicar estas diferencias en la enseñanza del trabajo de campo es la orientación relativamente más grupal de la antropología mexicana y el enfoque más individual de la antropología de Estados Unidos. Aunque ambas antropologías incluyen tradiciones tanto de trabajo en grupo como individual, considero que a nivel general hay una distinción. Me aventuraría a decir que hoy existen pocos proyectos colectivos que involucren a estudiantes de posgrado en la antropología sociocultural de Estados Unidos. A menudo, los estudiantes trabajan en los mismos sitios o en los mismos temas que sus tutores, pero no al mismo tiempo y no en el mismo proyecto. Aun más, raramente hacen trabajo de campo o publican juntos. En México, el número de proyectos colectivos ha disminuido, pero sigue siendo una práctica común. Esa orientación más individual en Estados Unidos puede explicarse en parte por el factor geográfico mencionado arriba, pero en mi opinión también es alentado por una lógica cultural dominante dentro de la disciplina y en la sociedad estadounidense en su conjunto que valora sobre todo los logros individuales, la originalidad y la novedad. Estos valores están ligados con un fuerte énfasis en la propiedad individual: ${ }^{2}$ cada antropólogo, incluyendo a los alumnos, tiene su propio tema, datos y lugar, los cuales deben ser respetados por los demás. La

\footnotetext{
${ }^{1}$ Los profesores y los estudiantes estadounidenses están juntos en el campo en el contexto de los field schools que algunas universidades de Estados Unidos tienen en países subdesarrollados. Nunca he participado en una, pero tengo la idea de que tienen más que ver con proporcionar una experiencia culturalmente distinta que con aprender métodos y técnicas de la investigación etnográfica. Además, se enfocan a estudiantes de licenciatura, no de posgrado.

${ }^{2}$ Estoy en deuda con Marisol Pérez Lizaur por esta interpretación.
}

importancia que se otorga a la propiedad individual tiene efectos en la forma en que se practica y se enseña la antropología. Por ejemplo, desalienta a los profesores de publicar con sus estudiantes por el respeto a la propiedad intelectual de éstos. Esta protección de los derechos de propiedad intelectual de los estudiantes es, sin duda, algo bueno, pero pienso que el énfasis en ello en Estados Unidos merece reflexionarse no sólo como cuestión de "racionalidad" sino también como un producto histórico-cultural.

A mi parecer, en México al menos tres factores explican la relevancia del trabajo colectivo. Primero, en la historia de la antropología mexicana, comparada con la de Estados Unidos, la tradición de la investigación colectiva, en parte atribuible a la influencia de Palerm, ocupa un rol más dominante. Podría decirse que la imagen clásica de la antropología mexicana no es la del investigador solitario que llega a un sitio aislado, sino la de un equipo que trabaja en conjunto, bajo la supervisión de un líder o líderes y que lleva a cabo la investigación en una región entera y no en un solo lugar. Segundo, en años recientes las instituciones del gobierno mexicano y algunas administraciones universitarias han intentado imponer la investigación colectiva, en particular la colaboración laboral entre profesores y estudiantes. Esto es en parte un producto de la imposición universal e indiscriminada de un modelo de la ciencia dura, en el cual se espera que los estudiantes de posgrado trabajen en los proyectos de sus tutores y publiquen con ellos como autores secundarios. También puede deberse a una pretensión de asegurar la productividad académica en tiempos de austeridad. El financiamiento de proyectos colectivos responsabiliza a los profesores de la productividad de sus alumnos de posgrado y a los más expertos investigadores de la productividad de sus colegas menos experimentados. ${ }^{3}$ Tercero, esta tendencia hacia la investigación colectiva refleja la tradición jerárquica y corporativista de México, en la cual las personas

\footnotetext{
${ }^{3}$ Estoy en deuda con Óscar Martínez por esta observación.
} 
están acostumbradas a relacionarse entre sí en los entornos institucionales mediante lazos patróncliente. Mientras muchos de mis colegas en México rechazan esta tradición y alientan a sus alumnos a ejercer la propia iniciativa y creatividad, la reproducción parcial de esta dinámica permanece inalterable no sólo porque se mantiene en la expectativa de muchos estudiantes, sino también porque en una gran proporción de los casos las oportunidades para publicar y el mercado laboral dependen más de los contactos personales e institucionales que de los logros individuales.

\section{CONCLUSIÓN: OPORTUNIDADES, RETOS Y AMENAZAS}

La tendencia hacia el trabajo más colectivo que surge de estos factores reporta varias ventajas importantes. Por ejemplo, ofrece oportunidades a los estudiantes de trabajar más de cerca y aprender de sus profesores y fomenta la colaboración sobre la competencia. Sin embargo, al mismo tiempo los pone en mayor riesgo de abuso debido a su posición dependiente y a la potencial falta de límites claros y firmes entre profesores y estudiantes y su trabajo respectivo. Podría decirse que mientras la antropología de Estados Unidos se caracteriza por un exceso de individualismo, quizá su contraparte mexicana adolezca de su falta. Irónicamente, las presiones para trabajar de manera colectiva en México no implican que hayamos escapado al aumento de evaluaciones cuantitativas individuales de nuestra producción académica. Como resultado, los profesores, preocupados por su propia producción, han empezado a cuestionar si es práctico darse tiempo para formar a los alumnos en el campo, aunque el hecho de que los profesores puedan hacer su propia investigación mientras capacitan a los estudiantes mitiga esta tensión. Entre tanto, la presión sobre los programas del Padrón Nacional de Posgrado del Consejo Nacional de Ciencia y Tecnología (Conacyt) de graduar a los alumnos de maestría en dos años y medio y a los de doctorado en cuatro y medio desalienta a dedicar parte de este tiempo limitado a una formación en campo que no constituye un avance directo en el proyecto de tesis del estudiante. Este énfasis en la eficiencia terminal ha llevado a una tensión no resuelta entre tratar la práctica de campo como un ejercicio de formación o como una oportunidad para obtener un adelanto en la investigación para la tesis.

El futuro de la práctica en la UIA, además, ha sido amenazado recientemente por cortes al presupuesto a nivel institucional. En dos ocasiones, la administración de la Universidad ha puesto en venta la estación de campo en Tepetlaoxtoc. En ambas ocasiones los responsables del Programa de Posgrado en Antropología Social lograron convencer a la administración de no venderla, pero no está claro qué sucederá la próxima vez. El Programa aún cuenta con fondos para los otros gastos de la práctica de campo, aunque enseñar a los estudiantes en un aula de la Universidad sería, sin duda, más económico. En suma, presento este ejemplo de la enseñanza del trabajo de campo en el campo de la UIA como una contribución a la discusión transnacional y comparativa sobre la formación de los alumnos de posgrado en la antropología social. Espero que este ejemplo proporcione nuevas, y no tan nuevas, ideas para mejorar la formación de los alumnos y para pensar, en forma más general, el futuro de la enseñanza de la disciplina. También creo que tiene el potencial de elevar nuestra conciencia sobre la manera en que los métodos de formación se ven influidos por factores sociales y culturales de los que a menudo no estamos conscientes en el entorno familiar y en apariencia culturalmente neutral de la academia. 